\title{
Development of polymorphic EST microsatellite markers for the sand fly, Phlebotomus papatasi (Diptera: Psychodidae)
}

Omar Hamarsheh ${ }^{1,11^{*}}$ (D), Mehmet Karakuş ${ }^{2}$, Kifaya Azmi $^{3}$, Kaouther Jaouadi ${ }^{4}$, Mohammad Reza Yaghoobi-Ershadi $^{5}$, Andreas Krüger ${ }^{6}$, Ahmad Amro ${ }^{7}$, Mohamed Amin Kenawy ${ }^{8}$, Mostafa Ramadhan Dokhan ${ }^{9}$, Ziad Abdeen ${ }^{10}$ and Mary Ann McDowell ${ }^{11}$

\begin{abstract}
Background: Phlebotomus papatasi is a widely distributed sand fly species in different tropical and sub-tropical regions including the Middle East and North Africa. It is considered an important vector that transmits Leishmania major parasites, the causative agents of cutaneous leishmaniasis. The development of microsatellite markers for this sand fly vector is of high interest to understand its population structure and to monitor its geographic dispersal.

Results: Fourteen polymorphic microsatellite markers were developed with simple di-, tri- and tetra-nucleotide repeats. The $F$ statistics calculated for the 14 markers revealed high genetic diversity; expected heterozygosity (He) ranged from 0.407 to 0.767 , while observed heterozygosity $(\mathrm{Ho})$ was lower and ranged from 0.083 to 0.514 . The number of alleles sampled fall in the range of 9-29. Three out of 14 markers deviated from Hardy-Weinberg expectations, no significant linkage disequilibrium was detected and high values for inbreeding coefficient $\left(F_{I S}\right)$ were likely due to inbreeding.

Conclusions: The development of these functional microsatellites enable a high resolution of $P$. papatasi populations. It opens a path for researchers to perform multi locus-based population genetic structure analyses, and comparative mapping, a part of the efforts to uncover the population dynamics of this vector, which is an important global strategy for understanding the epidemiology and control of leishmaniasis.
\end{abstract}

Keywords: Phlebotomus papatasi, Leishmania major, Microsatellites, Population structure

\section{Background}

Phlebotomus papatasi sand flies are vectors of Leishmania major parasites: the causative agents of cutaneous leishmaniasis in the Middle East and North Africa. The wide geographical range and the extensive use of insecticides, climate change, wars and natural catastrophes could affect population dynamics of vectors of infectious diseases [1-6]. Like most other sand flies, P. papatasi has received little attention by population geneticists;

\footnotetext{
* Correspondence: ohamarsheh@staff.alquds.edu

'Department of Biological Sciences, Faculty of Science and Technology,

Al-Quds University, Jerusalem, Palestine

${ }^{11}$ Department of Biological Sciences, Eck Institute for Global Health, Galvin

Life Science, University of Notre Dame, Notre Dame, IN 46656, USA

Full list of author information is available at the end of the article
}

molecular genetic studies on this species using various markers were documented [7-10] and no new microsatellites have been developed, except five polymorphic markers developed by our group in 2006 [11-13].

Due to high polymorphism information content and fast mutation rate, microsatellites have been used with success in the past for population analysis of various insects and sand flies including P. papatasi [13-21]. Like other nuclear DNA markers, microsatellites found in expressed sequence tags (ESTs) are of great value as they represent a set of functional markers. High mutation rates and simple Mendelian inheritance of these loci make them appropriate for investigations on population dynamics, breeding patterns and phylogeny [22, 23]. 
Although selection can be expected to be operating on a small percentage of EST markers, this drawback can be largely overcome by the use of a sufficient number of markers. On the other hand, markers proved to be under selection and non-neutral should be removed from the analysis.

Research based on EST analysis suggested that the frequency of microsatellites in some organisms is greater than was expected, had a reduced occurrence of null alleles, and had high transferability to other species [24, 25]. In this study we describe the identification of a new panel of 14 polymorphic microsatellites based on our previously mined $P$. papatasi EST simple sequence repeats [16].

\section{Methods}

One hundred and one flies originating from 19 locations in six countries have been analyzed, including two laboratory colonies and one field population from Egypt, one laboratory colony and seven field populations from Turkey, two field populations from Tunisia, three field populations from Iran, two field populations from Afghanistan, and one laboratory colony from Cyprus. DNA was extracted from five individual flies in each population using a DNA extraction kit (Invitrogen, Carlsbad, CA, USA), following the manufacturer's instructions. The EST primers were selected from a list of EST primers which has been mined previously by our group [16] and based on the following criteria: number of tandem repeat motifs $\geq 5$, no compound motifs were used, and loci were selected from different contigs to avoid linkage disequilibrium.

The PCR reactions were carried out in a $25 \mu \mathrm{l}$ reaction mixture containing $2.5 \mu \mathrm{l} \mathrm{10 \times} \mathrm{PCR} \mathrm{buffer,} 0.5 \mu \mathrm{l}$ dNTP mixture, $0.15 \mu \mathrm{l}$ of TaKaRa Taq, $1.2 \mu \mathrm{l}$ of template DNA, and $0.5 \mu \mathrm{M}$ of each primer. For PCR amplification, DNA was denatured at $94{ }^{\circ} \mathrm{C}$ for $5 \mathrm{~min}$ followed by 35 cycles $\left(94{ }^{\circ} \mathrm{C}\right.$ for $45 \mathrm{~s}$, annealing for $40 \mathrm{~s}, 72{ }^{\circ} \mathrm{C}$ for $45 \mathrm{~s}$ ), and a final extension at $72{ }^{\circ} \mathrm{C}$ for $7 \mathrm{~min}$. Polymorphisms were evaluated by separating PCR products on high resolution 3.5\% MetaPhore agarose gel (Lonza, Rockland, ME, USA). For accurate sizing of the polymorphic PCR products, the forward primers were labeled with 5 - fluorescent dyes (D2-D4). The PCR products were then analyzed using the automated $\mathrm{CEQ}^{\mathrm{TM}} 8000$ sequencer (Beckman Coulter, Fullerton, CA, USA) and the fragment sizes were analyzed using its fragment analysis tool. Estimates of heterozygosity, inbreeding coefficient $\left(F_{\mathrm{IS}}\right)$, and allele counts were completed using the software package FSTAT version 2.9.3.2 [26]. As null alleles can overestimate $F_{\text {IS }}$ values, the Bayesian based individual inbreeding model (IIM) implemented in the program INEST $2.0[27,28]$ was used to simultaneously estimate the presence of null alleles and inbreeding coefficients. INEST was run using nfb (null alleles, in breeding coefficients, and genotyping failures) and $\mathrm{nb}$ (null alleles and genotyping failures) models to detect the existence of inbreeding effects in our dataset. The number of cycles (MCHC iterations) was set to 500,000 and 'burn-in' was 50,000. Tests for Hardy-Weinberg

Table 1 Primer sequences and locus characteristics

\begin{tabular}{|c|c|c|c|c|c|c|c|}
\hline Locus & Accession no. & Repeat & $\begin{array}{l}\text { Fragment } \\
\text { size (bp) }\end{array}$ & Forward $\left(5^{\prime}-3^{\prime}\right)^{a}$ & Reverse (5'-3') & $\operatorname{Tm}\left({ }^{\circ} \mathrm{C}\right)^{\mathrm{b}}$ & Gene $^{c}$ \\
\hline PPEST 2 & FG10856.1 & $(G C A) 13$ & 141 & D2-TGTCAATAGTGGCTCAATGCTC & ATTAGTCGTTTATCCTTCCCCG & 60 & PPAI004876-PA \\
\hline PPEST 10 & FG117371.1 & (TC) 12 & 236 & D2-ACTGAATCTTCTGCTTTCTCCATTC & TAAGGGAAGGGGCGGAAC & 60 & PPAI001073-PA \\
\hline PPEST 11 & ES347986.1 & $(G A) 11$ & 162 & D4-GGTGGATACTTGTGACGACTGA & CCACTCAAACTAAACTGGAAAGC & 60 & PPAI005664-PA \\
\hline DEST 17 & FG1 16712.1 & $(\mathrm{TGC}) 9$ & 208 & D4-CTGTTCAGCAAAACGAGACG & TCCCAAGTACAAAGACGGAACT & 60 & PPAI008660-PA \\
\hline PPEST 33 & FG1 15100.1 & $(G A A) 15$ & 251 & D4-ATACTCCCTCAGAACTAGCCCC & TTCGTCTTCTTCTTCTTCCTCC & 60 & PPAI005234-PA \\
\hline PPEST 34 & EY215687.1 & $(\mathrm{AAAG}) 5$ & 137 & D3-CACCTACAGAGATGCTGGATTG & GGGCTAAAATGTGTCTTGACTTG & 62 & PPAI003756-PA \\
\hline PPEST 40 & FG1 14532.1 & $(\mathrm{AG}) 5$ & 322 & D2-TCCCAAGGCTATTAAGTCTGGT & GGCTATCGTGCAATTTTCTTCT & 62 & PPAI005295-PA \\
\hline PEEST43 & EX474024.1 & $(\mathrm{CT}) 8$ & 228 & D3-AAAAGAGATITCGGGGAAGG & GATTGTTGAAGGAGTGAAAGGG & 62 & PPAI008911-PA \\
\hline PPEST49 & FG1 14549.1 & $(\mathrm{AG}) 10$ & 171 & D2-AAACAGCTTCAATCGCTCTGAC & СTCACTCACTCTCCCTTCGTCT & 60 & PPAI001715-PA \\
\hline PPEST68B & ES349040.1 & $(\mathrm{CA}) 6$ & 195 & D3-TGATTTCACCCTTGTGTTTCC & TGTGGCAACTTATTTACATCCC & 57.1 & PPAI009554-PA \\
\hline PPEST73 & EY213549.1 & $(\mathrm{GA}) 7$ & 244 & D3-CCAACAATCTCCTCTCTATCGC & CCCTCAAGCTAACAAACACACA & 57.1 & PPAI009131-PA \\
\hline PPEST75 & EY203801.1 & $(\mathrm{GTT}) 5$ & 237 & D4-TTGTCAGGAAAGGAGTTGTTCA & GTATGCAGCTCTCCCAGAAGAT & 55.5 & PPAI006509-PA \\
\hline PPEST77 & EY204214.1 & $(\mathrm{GA}) 8$ & 181 & D2-AATCTCAATCTGGGCAATGTGT & ACCTTCTCTGTAAATTCCCTTGG & 57.1 & PPAI004565-PA \\
\hline PPEST85 & EY208767.1 & $(\mathrm{GA}) 9$ & 244 & D4-GTTGAAGGGAAATTGTGAGGAT & ATGGACATTTGTGGACTCTGC & 60 & PPAI003566-PA \\
\hline
\end{tabular}

${ }^{\mathrm{a}}$ Forward primer labeled with either D2, D3, or D4 fluorescent dye at 5' end

${ }^{\mathrm{b}}$ Annealing temperature

'Gene code based on full P. papatasi genome assembly (available at www.vectorbase.org 
equilibrium and linkage disequilibrium were done using the GenAlEx package [29].

\section{Results and discussion}

Out of 721 potential microsatellites already mined in our previous work [16], 85 primer pairs were selected and optimized. Thirty-four primer pairs successfully amplified the target sequence and generated a single band of the correct size in preliminary screening using agarose gel electrophoresis. A total of 14 microsatellite markers were found polymorphic when tested on $P$. papatasi flies from different countries (Table 1 ).

The expected heterozygosity $(\mathrm{He})$ for all loci was relatively higher than observed heterozygosity $(\mathrm{Ho})$, ranging between $0.083-0.514$ (Table 2) suggesting a heterozygote deficiency, which has been reported previously for $P$. papatasi microsatellites [11]. The gap between $H o$ and $H$ e values, suggests the presence of null alleles, isolation, genetic drift, population sub structuring (Wahlund effect) or inbreeding [30]. However, this gap may be due to high inbreeding as revealed by relatively positive high $F_{\text {IS }}$ values calculated by FSTAT and INEST 2.0 programs.

The deviance information criterion (DIC) calculated from the "nfb" model gave a lower value $(23,612.759)$ than the "nb" model $(24,696.659)$ supporting the inbreeding model and its strong effect (Additional file 1: Table S1 and Additional file 2: Table S2) rather than the null allele model.

Table 2 Summary of descriptive statistics of P. papatasi microsatellite markers

\begin{tabular}{llllll}
\hline Locus & $H^{\mathrm{a}}$ & $H e^{\mathrm{b}}$ & $F_{\text {IS FSTAT/ INEST }}^{c}$ & No. of alleles & HWE $^{\mathrm{d}}$ \\
\hline PPEST33 & 0.102 & 0.572 & $0.842 / 0.808$ & 29 & 0.020 \\
PPEST77 & 0.296 & 0.697 & $0.557 / 0.573$ & 11 & 0.339 \\
PPEST2 & 0.083 & 0.407 & $0.802 / 0.666$ & 9 & 0.113 \\
PPEST75 & 0.192 & 0.481 & $0.600 / 0.595$ & 9 & 0.120 \\
PPEST11 & 0.307 & 0.64 & $0.565 / 0.526$ & 10 & 0.125 \\
PPEST73 & 0.218 & 0.489 & $0.552 / 0.632$ & 10 & $0.019^{*}$ \\
PPEST68 & 0.415 & 0.767 & $0.464 / 0.466$ & 17 & 0.598 \\
PPEST85 & 0.182 & 0.570 & $0.657 / 0.640$ & 15 & 0.132 \\
PPEST10 & 0.315 & 0.648 & $0.513 / 0.536$ & 13 & $0.020^{*}$ \\
PPEST34 & 0.514 & 0.75 & $0.315 / 0.328$ & 13 & 0.224 \\
PPEST17 & 0.337 & 0.544 & $0.360 / 0.456$ & 11 & 0.244 \\
PPEST40 & 0.376 & 0.649 & $0.435 / 0.521$ & 13 & 0.451 \\
PPEST49 & 0.219 & 0.517 & $0.580 / 0.630$ & 14 & 0.804 \\
PPEST43 & 0.278 & 0.651 & $0.598 / 0.589$ & 11 & $0.025^{*}$ \\
\hline
\end{tabular}

abserved heterozygosity

${ }^{b}$ Expected heterozygosity

'Wright Inbreeding coefficients $\left(F_{\mathrm{IS}}\right)$, calculated using both FSTAT and in INEST 2.0

${ }^{\mathrm{d}} P$-values of the exact test for Hardy-Weinberg equilibrium $\left({ }^{*} P<0.05\right.$ considered significant)
One limitation of using EST-SSRs is that they generally considered less polymorphic than other microsatellite marker types, but have the benefit of an efficient and economic method and reduced occurrence of null alleles because the DNA sequences flanking SSRs from transcribed regions are relatively stable [25]. Therefore, the markers described here are very promising and can be used with confidence for population structure studies of this sand fly vector.

A few loci, markers PPEST73, PPEST10, and PPEST43, deviated significantly from Hardy-Weinberg expectations, and therefore provide caution of the utilization of these markers. None of the loci were in linkage disequilibrium (LD); all genotypic disequilibrium comparisons showed $P$-values above the 5\% nominal level (0.00055). The number of alleles per locus ranged from 9 to 29 alleles, with the higher number of alleles observed in our study being likely due to the higher resolution of fluorescence-based genotyping as well as the inclusion of many field caught flies. These markers may have transferability among other species. However, tests for transferability should be completed on all sand fly species to extend the usefulness of these markers for interspecies studies.

Mining EST sequences is an effective strategy to identify functional microsatellites in P. papatasi sand flies. The polymorphic microsatellite markers discovered in this study will be useful for further population structure analysis, comparative mapping between populations or species, and determining the changes occurred as a result of selection.

\section{Conclusions}

The decreased expenses of development, and lower frequency of null alleles are significant benefits of EST microsatellites, they considered valuable and appropriate markers for future population genetic studies and comparative mapping in $P$. papatasi. Transferability evaluation should be completed, in order to extend the benefits of these markers to other sand fly species.

\section{Additional files}

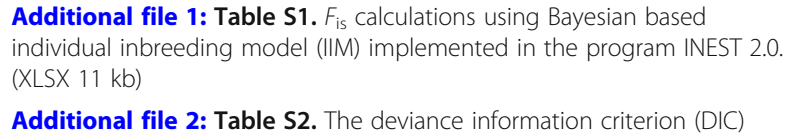

Additional file 2: Table S2. The deviance information criterion (DIC) comparison results between "nb" and "nbf" models. (XLSX 35 kb)

\footnotetext{
Abbreviations

DIC: deviance information criterion; EST: expressed sequence tags; $F_{15}$ : Wright's inbreeding coefficient; He: expected heterozygosity; Ho: observed heterozygosity; HWE: Hardy-Weinberg equilibrium; LD: linkage disequilibrium; SSR: simple sequence repeat
} 


\section{Acknowledgements}

Not applicable.

\section{Funding}

This work was supported by a grant from the Eck Institute for Global Health at the University of Notre Dame and travel grants awarded to Omar Hamarsheh from Fulbright Association and Zamallah Academic Development Programme.

\section{Availability of data and materials}

The datasets generated during and/or analyzed during the current study are not publicly available since these genotypic data together with other datasets will be used for further population structure analysis, but are available from the corresponding author upon reasonable request.

\section{Authors' contributions}

$\mathrm{OH}$ and $\mathrm{MM}$ designed the study, carried on the experimental work and drafted the manuscript. KA, MK, KJ, AA, AK, RY, MAK, ZA and MD carried on field work and provided sand flies for this study. All authors read and approved the final manuscript.

\section{Ethics approval and consent to participate}

Not applicable.

\section{Consent for publication}

Not applicable.

\section{Competing interests}

The authors declare that they have no competing interests.

\section{Publisher's Note}

Springer Nature remains neutral with regard to jurisdictional claims in published maps and institutional affiliations.

\section{Author details}

${ }^{1}$ Department of Biological Sciences, Faculty of Science and Technology, Al-Quds University, Jerusalem, Palestine. ${ }^{2}$ Department of Parasitology, Faculty of Medicine, Ege University, Izmir, Turkey. ${ }^{3}$ Department of Biochemistry and Molecular Biology, Faculty of Medicine, Al-Quds University, Jerusalem, Palestine. ${ }^{4}$ Department of Medical Epidemiology, Laboratory of Transmission, Control and Immunobiology of Infections (LR11IPT02) Institut Pasteur de Tunis, 13 Place Pasteur BP-74, 1002 Tunis-Belvedere, Tunisia. ${ }^{5}$ Department of Medical Entomology \& Vector Control, School of Public Health, Tehran University of Medical Sciences, Tehran, Iran. ${ }^{6}$ Department of Tropical Medicine, Military Hospital Hamburg, Bernhard-Nocht-Straße 74, 20359 Hamburg, Germany. 7 Faculty of Pharmacy, Al-Quds University, Jerusalem, Palestine. ${ }^{8}$ Department of Entomology, Faculty of Science, Ain Shams University, Abbassia, Cairo 11566, Egypt. ${ }^{9}$ Department of Zoology, Faculty of Science, University of Sabratha, Sabratha, Libya. ${ }^{10}$ Faculty of Medicine, Al-Quds University, Jerusalem, Palestine. ${ }^{11}$ Department of Biological Sciences, Eck Institute for Global Health, Galvin Life Science, University of Notre Dame, Notre Dame, IN 46656, USA

\section{Received: 21 April 2017 Accepted: 5 March 2018}

\section{Published online: 09 March 2018}

\section{References}

1. Dhiman RC, Mittal PK. A note on susceptibility status of Phlebotomus papatasi (Scopoli) populations to insecticides. J Commun Disord. 2000;32:65-6.

2. Lindahl JF, Grace D. The consequences of human actions on risks for infectious diseases: a review. Infect Ecol Epidemiol. 2015;5:30048.

3. Du R, Hotez PJ, Al-Salem WS, Acosta-Serrano A. Old World cutaneous leishmaniasis and refugee crises in the Middle East and North Africa. PLoS Negl Trop Dis. 2016;10(5):e0004545.

4. Pener $\mathrm{H}$, Wilamovsky A. Base-line susceptibility of Phlebotomus papatasi to insecticides. Med Vet Entomol. 1987:1:147-9.

5. Alawieh A, Musharrafieh U, Jaber A, Berry A, Ghosn N, Bizri AR. Revisiting leishmaniasis in the time of war: the Syrian conflict and the Lebanese outbreak. Int J Infect Dis. 2014;29:115-9.
6. Inci R, Ozturk P, Mulayim MK, Ozyurt K, Alatas ET, Inci MF. Effect of the Syrian Civil War on prevalence of cutaneous leishmaniasis in southeastern Anatolia, Turkey. Med Sci Monit. 2015;21:2100-4.

7. Depaquit J, Lienard E, Verzeaux-Griffon A, Ferte $H$, Bounamous A, Gantier JC, et al. Molecular homogeneity in diverse geographical populations of Phlebotomus papatasi (Diptera, Psychodidae) inferred from ND4 mtDNA and ITS2 rDNA epidemiological consequences. Infect Genet Evol. 2008;8(2):159-70.

8. Parvizi P, Ready PD. Molecular investigation of the population differentiation of Phlebotomus papatasi, important vector of Leishmania major, in different habitats and regions of Iran. Iran Biomed J. 2006; 10(2):69-77.

9. Ready PD. Biology of phlebotomine sand flies as vectors of disease agents. Annu Rev Entomol. 2013;58:227-50.

10. Esseghir S, Ready PD, Killick-Kendrick R, Ben-Ismail R. Mitochondrial haplotypes and phylogeography of Phlebotomus vectors of Leishmania major. Insect Mol Biol. 1997;6(3):211-25.

11. Hamarsheh O, Presber W, Abdeen Z, Sawalha S, Al-Lahem A, Schoenian G. Isolation and characterization of microsatellite loci in the sand fly Phlebotomus papatasi (Diptera: Psychodidae). Mol Ecol Notes. 2006;6(3): 826-8.

12. Hamarsheh $\mathrm{O}$, Presber W, Al-Jawabreh A, Abdeen Z, Amro A, Schonian G. Molecular markers for Phlebotomus papatasi (Diptera: Psychodidae) and their usefulness for population genetic analysis. Trans R Soc Trop Med Hyg. 2009;103(11):1085-6.

13. Hamarsheh O, Presber W, Yaghoobi-Ershadi MR, Amro A, Al-Jawabreh A, Sawalha S, et al. Population structure and geographical subdivision of the Leishmania major vector Phlebotomus papatasi as revealed by microsatellite variation. Med Vet Entomol. 2009;23(1):69-77.

14. Han B, Wang C, Tang Z, Ren Y, Li Y, Zhang D, et al. Genome-wide analysis of microsatellite markers based on sequenced database in Chinese spring wheat (Triticum aestivum L.). PLoS One. 2015;10(11):e0141540.

15. Dettori MT, Micali S, Giovinazzi J, Scalabrin S, Verde I, Cipriani G. Mining microsatellites in the peach genome: development of new long-core SSR markers for genetic analyses in five Prunus species. Springerplus. 2015:10(4):337.

16. Hamarsheh O, Amro A. Characterization of simple sequence repeats (SSRS) from Phlebotomus papatasi (Diptera: Psychodidae) expressed sequence tags (ESTs). Parasit Vectors. 2011;4:189.

17. Van't Hof AE, Brakefield PM, Saccheri IJ, Zwaan BJ. Evolutionary dynamics of multilocus microsatellite arrangements in the genome of the butterfly Bicyclus anynana, with implications for other Lepidoptera. Heredity. 2007;98(5):320-8.

18. Zhang L, Ma Y, Xu J. Genetic differentiation between sandfly populations of Phlebotomus chinensis and Phlebotomus sichuanensis (Diptera: Psychodidae) in China inferred by microsatellites. Parasit Vectors. 2013;6:115.

19. Neal AT, Ross MS, Schall JJ, Vardo-Zalik AM. Genetic differentiation over a small spatial scale of the sand fly Lutzomyia vexator (Diptera: Psychodidae). Parasit Vectors. 2016;9:550.

20. Aransay AM, Malarky G, Ready PD. Isolation (with enrichment) and characterization of trinucleotide microsatellites from Phlebotomus perniciosus, a vector of Leishmania infantum. Mol Ecol Notes. 2001;1(3):176-8.

21. Watts PC, Boyland E, Noyes HA, Maingon R, Kemp SJ. Polymorphic dinucleotide microsatellite loci in the sandfly Lutzomyia longipalpis (Diptera: Phlebotominae). Mol Ecol Notes. 2002;2(1):62-4.

22. Seyfert AL, Cristescu ME, Frisse L, Schaack S, Thomas WK, Lynch M. The rate and spectrum of microsatellite mutation in Caenorhabditis elegans and Daphnia pulex. Genetics. 2008;178(4):2113-21.

23. Ellegren $\mathrm{H}$. Microsatellites: simple sequences with complex evolution. Nat Rev Genet. 2004;5(6):435-45.

24. Morgante M, Hanafey M, Powell W. Microsatellites are preferentially associated with nonrepetitive DNA in plant genomes. Nat Genet. 2002; 30(2):194-200.

25. Kim KS, Ratcliffe ST, French BW, Liu L, Sappington TW. Utility of ESTderived SSRs as population genetics markers in a beetle. J Hered. 2008; 99(2):112-24

26. Goudet J. FSTAT v1.2. A computer program to calculate F-statistics.J Heredity. 1995;86:485-6.

27. Chybicki IJ, Burczyk J. Simultaneous estimation of null alleles and inbreeding coefficients. J Here. 2009;100(1):106-13. 
28. Campagne $P$, Smouse $P$, Varouchas $G$, Silvain JF, Leru B. Comparing the van Oosterhout and Chybicki-Burczyk methods of estimating null allele frequencies for inbred populations. Mol Ecol Resour. 2012;12(6):975-82.

29. Peakall R, Smouse PE. GenAlEx 6.5: genetic analysis in Excel. Population genetic software for teaching and research - an update. Bioinformatics. 2012;28(19):2537-9.

30. Callen DF, Thompson AD, Shen Y, Phillips HA, Richards RI, Mulley JC, Sutherland GR. Incidence and origin of "null" alleles in the (AC)n microsatellite markers. Am J Hum Genet. 1993;2(5):922-7.

Submit your next manuscript to BioMed Central and we will help you at every step:

- We accept pre-submission inquiries

- Our selector tool helps you to find the most relevant journal

- We provide round the clock customer support

- Convenient online submission

- Thorough peer review

- Inclusion in PubMed and all major indexing services

- Maximum visibility for your research

Submit your manuscript at www.biomedcentral.com/submit 